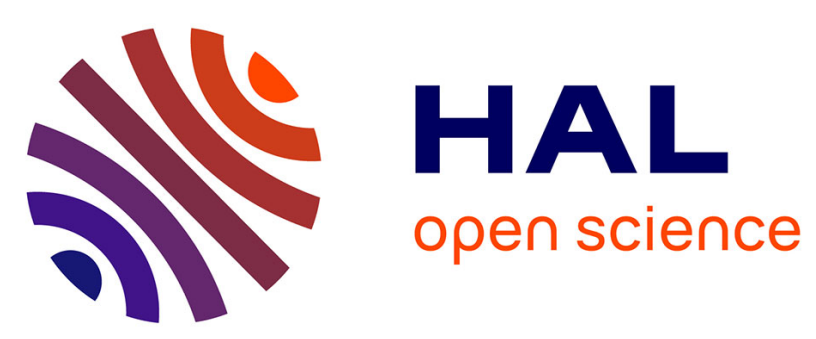

\title{
Food intake and energy expenditure are increased in high-fat-sensitive but not in high-carbohydrate-sensitive obesity-prone rats.
}

Dalila D. Azzout-Marniche, Catherine Chaumontet, Nachiket A Nadkarni, Julien Piedcoq, Gilles Fromentin, Daniel Tomé, Patrick Even

\section{To cite this version:}

Dalila D. Azzout-Marniche, Catherine Chaumontet, Nachiket A Nadkarni, Julien Piedcoq, Gilles Fromentin, et al.. Food intake and energy expenditure are increased in high-fat-sensitive but not in high-carbohydrate-sensitive obesity-prone rats.. AJP - Regulatory, Integrative and Comparative Physiology, 2014, 307 (3), pp.R299-309. 10.1152/ajpregu.00065.2014 . hal-01173413

\section{HAL Id: hal-01173413 \\ https://hal.science/hal-01173413}

Submitted on 28 May 2020

HAL is a multi-disciplinary open access archive for the deposit and dissemination of scientific research documents, whether they are published or not. The documents may come from teaching and research institutions in France or abroad, or from public or private research centers.
L'archive ouverte pluridisciplinaire HAL, est destinée au dépôt et à la diffusion de documents scientifiques de niveau recherche, publiés ou non, émanant des établissements d'enseignement et de recherche français ou étrangers, des laboratoires publics ou privés. 


\title{
Food intake and energy expenditure are increased in high-fat-sensitive but not
}

\section{in high-carbohydrate-sensitive obesity-prone rats}

\author{
Dalila Azzout-Marniche, ${ }^{1,2}$ Catherine Chaumontet, ${ }^{1,2}$ Nachiket A. Nadkarni, ${ }^{1,2}$ Julien Piedcoq, ${ }^{1,2}$ \\ Gilles Fromentin, ${ }^{1,2}$ Daniel Tomé, ${ }^{1,2}$ and Patrick C. Even ${ }^{1,2}$ \\ ${ }^{1}$ Institut National de la Recherche Agronomique, Centre de Recherche en Nutrition Humaine d'Ile-de-France (CRNH-IdF), \\ UMR 914 Nutrition Physiology and Ingestive Behavior, Paris, France; and ${ }^{2}$ AgroParisTech, CRNH-IdF, UMR 914 Nutrition \\ Physiology and Ingestive Behavior, Paris, France
}

Submitted 11 February 2014; accepted in final form 28 May 2014

\begin{abstract}
Azzout-Marniche D, Chaumontet C, Nadkarni NA, Piedcoq J, Fromentin G, Tomé D, Even PC. Food intake and energy expenditure are increased in high-fat-sensitive but not in high-carbohydrate-sensitive obesityprone rats. Am J Physiol Regul Integr Comp Physiol 307: R299-R309, 2014. First published June 4, 2014; doi:10.1152/ajpregu.00065.2014.—Obesityprone (OP) rodents are used as models of human obesity predisposition. The goal of the present study was to identify preexisting defects in energy expenditure components in OP rats. Two studies were performed. In the first one, male Wistar rats $(n=48)$ were fed a high-carbohydrate diet (HCD) for 3 wk and then a high-fat diet (HFD) for the next $3 \mathrm{wk}$. This study showed that adiposity gain under HCD was 2.9-fold larger in carbohydrate-sensitive (CS) than in carbohydrate-resistant (CR) rats, confirming the concept of "carbohydratesensitive" rats. Energy expenditure (EE), respiratory quotient (RQ), caloric intake (CI), and locomotor activity measured during HFD identified no differences in EE and RQ between fat-resistant (FR) and fat-sensitive (FS) rats, and indicated that obesity developed in FS rats only as the result of a larger CI not fully compensated by a parallel increase in EE. A specific pattern of spontaneous activity, characterized by reduced activity burst intensity, was identified in FS rats but not in CS ones. This mirrors a previous observation that under HCD, CS but not FS rats, exhibited bursts of activity of reduced intensity. In a second study, rats were fed a HFD for $3 \mathrm{wk}$, and the components of energy expenditure were examined by indirect calorimetry in 10 FR and $10 \mathrm{FS}$ rats. This study confirmed that a low basal EE, reduced thermic effect of feeding, defective postprandial energy partitioning, or a defective substrate utilization by the working muscle are not involved in the FS phenotype.
\end{abstract}

obesity-prone; energy balance; locomotor activity; indirect calorimetry; mRNA; blood parameters

GENETIC AND EPIGENETIC FACTORS make some individuals more sensitive than others to diet-induced obesity $(20,45)$. Outbred polygenetic rodents exhibit this variability in individual responses to diets $(30,50)$.

In rats, sensitivity to obesity has mainly been studied in response to a high-fat diet (HFD), and numerous differences between obesity-prone (OP) and obesity-resistant (OR) rats have been reported. After adaptation to a HFD and development of obesity, OP rats present intrinsic differences in norepinephrine metabolism (25), altered arcuate neuropeptide Y (NPY) expression (26), impairment in growth hormone secretion (24), leptin resistance $(29,31)$, defective counter-regulatory responses to insulin-induced hypoglycemia (46), lower interscapular brown adipose tissue mitochondrial uncoupling

Address for reprint requests and other correspondence: P. C. Even, AgroParisTech, CRNH-IdF, UMR914 Nutrition Physiology and Ingestive Behavior, F-75005 Paris, France (e-mail: patrick.even@agroparistech.fr). protein-1 content and proton conductance (18), and reduced dopamine levels in the nucleus accumbens (37). It is, however, difficult in these studies to conclude whether the observed differences are the cause or the consequence of the HFDinduced obesity. Studies conducted early after high-fat feeding are less numerous, but high plasma triglycerides (TG) $(11,23)$, elevated leptin, insulin, TG (11), tissue-specific changes in lipoprotein lipase $(11,36)$, and greater expression of the opioid enkephalin in various areas of the hypothalamus (7) early in response to high-fat (HF) feeding have been described as possible defects that may contribute to the sensitivity to HF feeding.

Large differences in adiposity gain can also be observed under a high-carbohydrate diet (HCD) in mice (51) and in rats $(15,34)$. Obesity under HC feeding develops more viscerally, and in our hands, only half of the rats prone to obesity under $\mathrm{HC}$ feeding are also prone to obesity under HF feeding and vice versa. Therefore, the mechanisms responsible for the predisposition to obesity under HC feeding are not necessarily the same as those that promote body fat gain under HF feeding. Accordingly, from now on, we divide OP rats into fat-sensitive (FS, as opposed to fat-resistant, FR) and carbohydrate-sensitive (CS, as opposed to carbohydrate-resistant, CR) rats. In search of metabolic and/or behavioral factors able to predict for fat or carbohydrate sensitivity, we previously reported that in young lean rats fed a standard $\mathrm{HCD}$, rats that would later demonstrate a sensitivity to a HFD had smaller meal size and larger meal number while rats that would later demonstrate a sensitivity to HCD had lower intensity of motor activity and higher ingestion speed (15). These parameters are, however, difficult to use as predictive factors to separate individuals because of significant overlap in individuals' data. In a following study, we performed a detailed analysis of the components of energy expenditure in FR/FS and CR/CS rats that revealed that FS rats did not exhibit any defect in any components of energy expenditure before being fed a HFD, but that CS rats had higher postmeal glucose oxidation and lower postmeal lipid oxidation than CR rats (34).

In previous studies $(15,34), \mathrm{CS}$ and FS rats were mainly studied while fed a HCD or during transition from a HCD to a HFD. The goal of the present study was to further evaluate body weight, body composition, components of energy expenditure, and feeding and activity patterns in CS and FS rats, more particularly after adaptation to HF feeding. In a first experiment, 48 rats were classified as CR or CS and FR or FS, according to the evolution of their adiposity measured by MRI during $3 \mathrm{wk}$ of $\mathrm{HC}$ feeding and then $3 \mathrm{wk}$ of $\mathrm{HF}$ feeding. During the third week of HF feeding, meal pattern, spontane- 
ous activity and total energy expenditure (TEE) were measured by indirect calorimetry coupled with recording of food intake and spontaneous activity. At the end of the study, the rats were killed; blood was taken for analysis of plasma circulating substrates; and tissues were sampled for quantification of mRNA expression for various genes in the liver, adipose tissue, and hypothalamus. In a second study, the components of energy expenditure were studied in detail under HF feeding in $10 \mathrm{FS}$ and 10 FR rats during a cycle of fasting and controlled refeeding, according to a procedure previously described $(14,34)$.

\section{MATERIALS AND METHODS}

The protocol conformed to the European legislation on the use of laboratory animals and was approved by the French Ethical Committee no. 11-027.

\section{Experiment 1}

Design. In a first experiment, male Wistar rats $(n=48)$ (Harlan), weighing $\sim 225 \mathrm{~g}$ (range 193-252 g) and 7 wk old, were delivered as six groups of eight, with groups arriving sequentially over a 9-mo period. Rats recovered in the laboratory for $1 \mathrm{wk}$ on a synthetic high-carbohydrate diet (HCD; Table 1) prior to any procedures. A 12:12-h light-dark cycle (lights on at 0800) was maintained throughout the experiment.

The design of the experiment was similar to that of Nadkarni et al. (34). The eight rats in each batch were scanned by MRI to measure their body fat content a week after arrival. Using these data, in general, for each batch, the two leanest and two fattest rats were kept, and the other four discarded from the study. The idea was to exploit the preexisting (small) differences in starting adiposity that develop after weaning to favor preselection of CR and CS rats [the maintenance diet of rats (chow) being a high-carbohydrate-low fat diet] and thus limit the number of rats excluded from the study. After the first MRI measurement, the rats were continued for another $3 \mathrm{wk}$ on the synthetic HCD and reanalyzed for body adiposity at the end of this period. At this point, $\mathrm{CR}$ and $\mathrm{CS}$ rats were segregated according to their increase in body adiposity during the HCD period. The rats were then fed for 3 wk on the HFD (Table 1) prior to another round of MRI. At this point, FR and FS rats were segregated on the basis of their gain in body adiposity during HFD. During the third week of HFD, the rats

Table 1. Macronutrient composition of the HCD and HFD

\begin{tabular}{lcc}
\hline \hline & HCD & HFD \\
\hline Weight content, g/kg & & \\
Milk proteins & 140.0 & 170.0 \\
Starch & 622.4 & 436.6 \\
Sucrose & 100.3 & 71.1 \\
Soy Oil & 40.0 & 225.0 \\
Minerals & 35.0 & 35.0 \\
Vitamins & 10.0 & 10.0 \\
Cellulose & 50.0 & 50.0 \\
Choline & 2.3 & 2.3 \\
Energy content, \% & & \\
Protein & 14.7 & 14.4 \\
Carbohydrate & 75.9 & 42.9 \\
Fat & 9.4 & 42.8 \\
Energy density, kJ/g & 15.95 & 19.82 \\
Food quotient & 0.946 & 0.847 \\
\hline
\end{tabular}

Macronutrients were prepared by the Atelier de Preparation des Aliments, INRA, Jouy en Josas, France. Energy density is computed assuming a metabolisable energy of $16.7 \mathrm{~kJ} / \mathrm{g}$ for carbohydrates and proteins and $37.7 \mathrm{~kJ} / \mathrm{g}$ for fat. Food quotient is computed assuming a quotient of oxidation of 1.0 for carbohydrates, 0.825 for proteins, and 0.70 for lipids. HCD, high-carbohydrate diet; HFD, high-fat diet. were housed for five consecutive days in metabolic cages with free access to the HFD for measurements of TEE, feeding behavior, and spontaneous motor activity. At the end of the study, the rats were anesthetized with halothane and killed by decapitation; then blood was collected. At this stage of the study a significant number of rats were too large to enter the MRI tunnel, or even if they could enter, their size caused image artifacts. Therefore, final body composition was measured by dissection and weighing of the main organs and tissues (16).

Calorimetry. MONITORING OF TEE, FEEDING BEHAVIOR AND SPONTANEOUS ACTIVITY UNDER HFD. Because of technical constraints, measurements of respiratory exchange, feeding pattern, and motor activity were performed in only 4 of the 6 groups of rats included in the study $(n=16)$. The goal was to obtain for each rat measures of meal pattern, spontaneous movement, TEE, and respiratory quotient (RQ) during HFD. All four rats in a group were housed at 1800 in individual metabolic cages equipped with a weighed food cup (sensitivity better than $0.05 \mathrm{~g}$ ) and with an activity platform placed below the cage (sensitivity better than $1 \mathrm{~g}$ ) (see Ref. 14 for details). For gas analysis, the cages were multiplexed, meaning all connected to the same gas analyzers. Thus $\dot{\mathrm{V}}_{2}$ and $\dot{\mathrm{V}}_{\mathrm{CO}_{2}}$ were measured for each cage during $2 \mathrm{~min}$ every $10 \mathrm{~min}(2 \mathrm{~min}$ for each cage plus $2 \mathrm{~min}$ on an empty cage-room air, to correct values for room $\mathrm{O}_{2} \%$ and $\mathrm{CO}_{2} \%$ ). Measurements were performed over 4 days. Day 1 in the metabolic cage was used for habituation. $\dot{\mathrm{V}}_{2}, \dot{\mathrm{V}}_{\mathrm{CO}_{2}}$, caloric intake $(\mathrm{CI})$, and spontaneous activity were measured on days 2,3 , and 4. Data were analyzed only when the feeding pattern could be confidently analyzed, in particular, when no food spillage was observed in the cage and/or suspected from the trace. This required exploiting only 2 out of the 3 days of recording in 10 out of the 16 rats. Metabolic rate was computed from $\dot{\mathrm{V}}_{2}$ and $\dot{\mathrm{V}}_{\mathrm{CO}_{2}}$, according to the Weir formula $(13,17)$. TEE, CI, and activity values reported are the results of the average values of 2 or 3 days of recording.

ADJUSTMENT OF TEE, CI, AND SPONTANEOUS ACTIVITY TO BODY SIZE AND COMPOSITION. As calorimetry sessions took place between the second round of MRI and dissection, the fat-free mass (FFM) and fat mass (FM) of the rats during the calorimetry sessions were estimated by linear extrapolation. The same technique was used in Nadkarni et al. (34) between the first two rounds of MRI. Here, as there, the technique was considered valid since the FFM+FM weights predicted by the extrapolation matched very well the actual measured body weight (BW) during calorimetry. The TEE and CI data were adjusted to per kilogram FFM and by a more recent attempt to take into account the metabolic activity of FM using (FFM $+0.2 \times$ FM) as the metabolically active body mass $(2,14)$. Spontaneous activity signal recorded from force transducers was normalized to per kilogram $\mathrm{BW}$ to take into account the fact that, for a given intensity of activity, the signal from the force transducers was proportional to the weight of the animal.

CALCULATION OF ENERGY BALANCE AND FOOD EFFICIENCY. Energy balance (EB) was computed from changes in body composition [assuming that $4.8 \mathrm{~kJ} / \mathrm{g}$ are fixed in lean tissues and $34 \mathrm{~kJ} / \mathrm{g}$ in adipose tissue (43)] for the whole 21 days of HFD feeding (EBMRI) and from differences between CI and TEE measured during the 5 days of food intake and calorimetry measurements (EBCalo). Food efficiency during calorimetry measurements was computed as the ratio of energy deposited to caloric intake $(\mathrm{kJ} / \mathrm{kJ})$.

Blood and tissue sampling. At the end of experiment 1, blood was collected from the trunk of the animal for measurements of TG, glycerol, ketone bodies, high-density lipoprotein (HDL), and cholesterol. Pieces of liver and epididymal fat were collected for assaying TG content and mRNA expression of genes, encoding key enzymes involved in different metabolic pathways, such as lipogenesis and glycerolipid biosynthesis (acetyl-CoA carboxylase, fatty acid synthase, glycerol-3-phosphate acyltransferase 1 mitochondrial), glycolysis (glucokinase), lipolysis and $\beta$-oxidation 
(monoglycerol lipase and carnitine acyltransferase 1, a liver isoform), lipoprotein trafficking (apolipoprotein A and E), and glycerol metabolism (glycerokinase) in the liver, acetyl CoA carboxylase, fatty acid synthase (FAS), leptin, and lipoprotein lipase in adipose tissue. The hypothalamus was dissected, put in TRIzol (Invitrogen) and snap frozen for measuring mRNA expression of neuropeptide Y, proopiomelanocortin, melanocortin 4 receptor, cocaine- and amphetamine-regulated transcript, Agoutirelated peptide, insulin receptor, leptin receptor (long cytoplasmic form), fat mass, and obesity-associated genes.

\section{Experiment 2}

Design. Forty male Wistar rats (Harlan), weighing $\sim 225 \mathrm{~g}$ (range 193-252 g) and 7 wk old, were delivered as five groups of eight, with groups arriving sequentially over a 6-mo period. Rats recovered in the laboratory for $1 \mathrm{wk}$ on the synthetic high-carbohydrate diet prior to being maintained on the HFD for 3 wk. A 12:12-h light-dark cycle (lights on at 0800) was maintained throughout.

Calorimetry. During the third week of HF feeding, since experiment 1 showed a strong correlation between BW gain and adiposity gain during HFD (see Fig. 3), rather than using MRI body composition measurements of adiposity, selection for further study was simply based on body weight gain; the two rats that gained the most and the two that gained the least weight in each group of eight were housed, in turn, for $24 \mathrm{~h}$ in a calorimetry device for a detailed analysis of the components of energy metabolism, as described previously $(14,34)$. In brief, the rats were housed in the cage at 1800 with water but were food-deprived overnight. Temperature in the cage was maintained at $26^{\circ} \mathrm{C}$ to reduce the cost of thermoregulation (14). The next day at

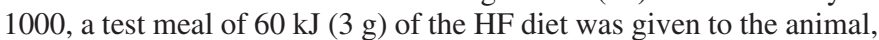
and data collection continued until 1700. As previously described (13, 14 ), the calorimetry system allowed separation of resting and activityrelated $\dot{\mathrm{V}}_{2}$ and $\dot{\mathrm{V}}_{\mathrm{CO}_{2}}$, thus precise itemizing of the various components of total energy expenditure. Briefly, the simultaneous recording of $\dot{\mathrm{V}}_{2}, \dot{\mathrm{V}}_{\mathrm{CO}_{2}}$, and the intensity of spontaneous activity at a high rate (1 point every 2 s) feeds a model of changes expected in $\dot{\mathrm{V}}_{2}$ and $\dot{\mathrm{V}} \mathrm{CO}_{2}$ values as a function of changes in the intensity of motor activity [cage volume and air flow are used to model the delay and damping of the response, and it is assumed that resting metabolic rate (RMR) and the cost of activity remain constant]. Model predictions are then compared with the actual measurements using a numerical filtering process (Kalman) that is tuned to maintain the model predictions close to the actual measurements. This leads to the computation over time of RMR and cost of activity values that fit the predictions of the model and the values actually recorded by the calorimetry device. This allows separation of resting and activity-related $\dot{\mathrm{V}}_{2}$ and $\dot{\mathrm{V}}_{\mathrm{CO}_{2}}$, thus precise itemizing of the various components of TEE. $\dot{\mathrm{V}}_{2}$ and $\dot{\mathrm{V}}_{\mathrm{CO}_{2}}(\mathrm{ml} / \mathrm{min})$ were converted to metabolic rate (Watts) using an adaptation of the Weir equation $\left[\left(16.3 \dot{\mathrm{V}}_{2}+4.57 \dot{\mathrm{V}}_{\mathrm{CO}_{2}}\right) / 60\right]$. RQ was computed as the ratio of $\dot{\mathrm{V}}_{\mathrm{CO}_{2}}$ to $\dot{\mathrm{V}}_{2}$. The thermic effect of feeding (TEF) was calculated from the increase in RMR induced by the test meal (and was, thus, free from any noise due to variations in spontaneous activity).

Body adiposity was measured by MRI the Monday that followed the measurements to control for the difference in adiposity between rats and to adjust energy expenditure to the metabolic body size $(\mathrm{FFM}+0.2 \times \mathrm{FM})(14)$. This same procedure applied in rats fed a HCD has been previously published (34).

Body composition by MRI analysis. Images were acquired on a 7T Bruker Pharmascan system (running Paravision 4) using a Bruker $50 \mathrm{~mm}$ ID tunable quadrature RF resonator (For details, see Ref. 34.) Images were registered, and then fat pads were segmented semiautomatically (by fuzzy c-means) in MIPAV 4.3.0. Adipose volume was converted to grams of FM on the assumption of a density of $0.9 \mathrm{~g} / \mathrm{cm}^{3}$, and FFM was determined by subtracting this from the weight of the rat on the day of the scan.

$R N A$ extraction and $R T-P C R$. Small pieces of liver and adipose tissue were taken and frozen in liquid nitrogen. To avoid RNA degradation, the hypothalamus was immediately extracted from the fresh brain by making an incision medial to the piriform lobes caudal to the optic chiasma and anterior to the cerebral crus to a depth of 2-3 mm and was put directly in TRIzol reagent and frozen in liquid nitrogen. This extraction was centered on the arcuate nucleus, which is the main site of expression of proopiomelanocortin (POMC) and NPY but also included part of the dorsal motor nucleus and lateral hypothalamus nuclei. Total RNA was extracted from liver, adipose tissue, and hypothalamus tissues with TRIzol reagent (Invitrogen, Breda, Netherlands). Concentrations of RNA samples were measured on a NanoDrop ND-1000 UV-Vis spectrophotometer. RNA integrity was checked by ethidium bromide staining. $0.4 \mu \mathrm{g}$ of total RNA in a final volume of $10 \mu \mathrm{l}$ was reverse transcribed using a high-capacity cDNA archive kit protocol (Applied Biosystems). Real-time PCR was performed to measure RNA expression using a ABI 7300 (Applied Biosystems using Power SYBR Green PCR Master Mix), as previously described (44). Reactions were performed as follows: denaturation for $10 \mathrm{~min}$ at $95^{\circ} \mathrm{C}, 40$ cycles at $95^{\circ} \mathrm{C}$ for $15 \mathrm{~s}$, followed by $1 \mathrm{~min}$ at $60^{\circ} \mathrm{C}$ (amplification). Negative controls (reactions without reverse transcriptase or RNA) were used to monitor for contamination. The efficiency was estimated using a series of five-fold dilutions of the sample and checked for each run. A melting curve was performed to check for the absence of contamination. The primer sequences of target genes are given Table 2. Gene expression was calculated as $2^{-\Delta C T}$. 18S RNA was used as the housekeeping gene in liver and adipose tissue, and RPL-13A RNA in hypothalamus.

Statistical analysis of postprocessed data. Results are reported as means \pm SE. Between-group comparisons were done by Student's $t$-tests in Excel. $P \leq 0.05$ was considered as significant. To select between sensitive and resistant rats in experiment 1 , we used the evolution of adiposity (defined as FM/BW) during the HCD (CR/ CS) and HFD (FR/FS) periods. For each period, the mean and the

Table 2. Primer sequences of target genes

\begin{tabular}{|c|c|c|}
\hline Gene & Upregulated & Downregulated \\
\hline$F A S$ & 5'-TGCTCCCAGCTGCAG-3' & 5'-GCCCGGTAGCTCTGGGTGTA-3' \\
\hline$A C C$ & 5'-CAACGCCTTCACACCACCTT-3' & 5'-AGCCCATTACTTCATCAAAGATCCT-3' \\
\hline Lept & 5'-CAAAGTCCAGGATGACACCAAA-3' & 5'-ATACCGACTGCGTGTGTGAAAT-3' \\
\hline$L P L$ & 5'-GGACTGAGGATGGCAAGCA-3' & 5'-GGCAGGGTGAAGGGAATGTT-3' \\
\hline$N P Y$ & 5'-TTTTCCTAGTTTCCCCCCACA-3' & 5'-CCTGGTGGTGGCATGCAT-3' \\
\hline POMC & 5'-AGGCCTTTCCCCTAGAGTTCAA-3' & 5'-GTCGGCCTTCTCGGTATCC-3' \\
\hline$M C 4 R$ & $5^{\prime}$-GGGAAAGCCACAAAAAACGA-3' & 5'-GGCGCTACTGAAAGCTCACTCT-3' \\
\hline$C A R T$ & $5^{\prime}$-CCGAGCCCTGGACATCTACTC-3' & 5'-AAATACTGACCAGCTCCTTCTCATG-3' \\
\hline$A G R P$ & 5'-TGGTGCCCTTGACCAAAGTT-3' & 5'-AATTTCTGCCCCCACAGATG-3' \\
\hline$I R$ & $5^{\prime}$-CCCAATGGCAACATCACACA-3' & 5'-CAGCTCGCTGTCCTCTGCTT-3' \\
\hline$O b-R$ & 5'-ACGATGCTTCACCACGTACCT-3' & $5^{\prime}$-GGAAGCCCCTGACGAAAAA-3' \\
\hline FTO & 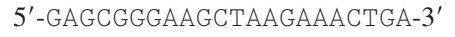 & $5^{\prime}$-GCCACTGCTGATAGAACTCATCA-3' \\
\hline
\end{tabular}


Student confidence interval (SCI) of adiposity gain at $\alpha=0.05$ were computed as $\mathrm{SD} \times 1.96 / \mathrm{SQRT}(n)$ (with $\mathrm{SD}$ for standard deviation and SQRT for square root). Resistant rats were defined as those whose adiposity gain was lower than the mean minus SCI, while sensitive rats were defined as those whose adiposity gain was higher than the mean plus SCI. During HCD, mean adiposity gain of all of the 24 rats was $3.74 \%$ with a $95 \%$ confidence interval of $0.76 \%$, and during HFD, mean adiposity gain was $6.34 \%$ with a $95 \%$ confidence interval of $0.86 \%$. Following this classification, and thanks to the initial preselection based on the initial adiposity of the rats, of the 24 rats included for the whole study, 10 rats that gained less than $2.99 \%$ adiposity were classified as CR, 8 that gained more than $4.50 \%$ were classified as CS, and only six were discarded as intermediate. Similarly, under a HFD, 10 rats that gained less than $5.48 \%$ were classified as FR, 7 that gained more than $7.20 \%$ were classified as FS, and 7 were discarded as intermediate.

For the sake of comparison between rats that varied largely in body size and composition, energy expenditure and caloric intake were adjusted to fat free mass (FFM) and active metabolic mass (AMM) computed as $(\mathrm{FFM}+0.2 \times \mathrm{FM})(14)$.

\section{RESULTS}

\section{Evolution of BW and Body Composition During HCD and HFD (Experiment 1)}

Individual responses of the rats to the HCD and HFD showed that CS rats were not necessarily FS and vice versa (Fig. 1A), with four out of the eight CS rats also being FS, and 6 out of the 10 CR being FR. However, a significant correlation between adiposity gain during HCD and HFD was observed (Fig. $1 B$ ). The 0.29 value of the $R^{2}$ coefficient indicates that sensitivity to one of the diets predicts only one-third of the sensitivity to the other diet.
During HCD, adiposity gain was $1.98 \% \pm 0.19$ in $\mathrm{CR}$ rats and $5.81 \% \pm 0.47$ in CS ones, meaning CS rats gained 2.9 times more adiposity than $\mathrm{CR}$ rats (Fig. 2A). This occurred without significant differences in BW gain, as the difference between CR and CS rats lay exclusively in FM gain, while the gain in FFM was much the same. In FS rats, adiposity, but also $\mathrm{BW}$ and FFM, all increased significantly more than in FR ones (Fig. 2B). During a HFD, adiposity gain was $4.52 \% \pm 0.22$ in FR rats and $9.11 \% \pm 0.53$ in FS ones (see Table 3 ). FS rats gained 2 times more fat, 1.6 times more weight and 1.2 times more FFM than FR rats (Fig. 2D). CS rats also gained more adiposity than CR ones during HFD but only 1.44 times more and the gains in BW and FFM were not significantly different (Fig. 2C).

Considering all rats together, the ratio of visceral to subcutaneous fat deposition was significantly larger under $\operatorname{HCD}(2.73 \pm 0.23)$ than under HFD $(1.39 \pm 0.13)(P<$ 0.001) (Fig. 2), but the potential adverse effect of such deposition in CS rats was moderated by the fact that under HCD they increased fat storage subcutaneously ( $~ 3$ times) more than viscerally ( $\sim 2$ times) so that the ratio of visceral to subcutaneous fat deposition was only $2.05 \pm 0.23$ vs. $3.17 \pm 0.35$ in $\mathrm{CR}$ rats $(P=0.02)$. However, considered in the long-term, such a specific visceral deposition of fat with a high-carbohydrate diet should be considered to be a potential aggravating factor compared with the more subcutaneous fat deposition observed under high-fat feeding.

Taken together, these results indicate that the accumulation of fat during HCD develops more viscerally than subcutaneously, and although adiposity gain was lower in absolute terms in CS rats, the difference in adiposity gain was relatively larger between CR and CS rats than between FR and FS rats. Because
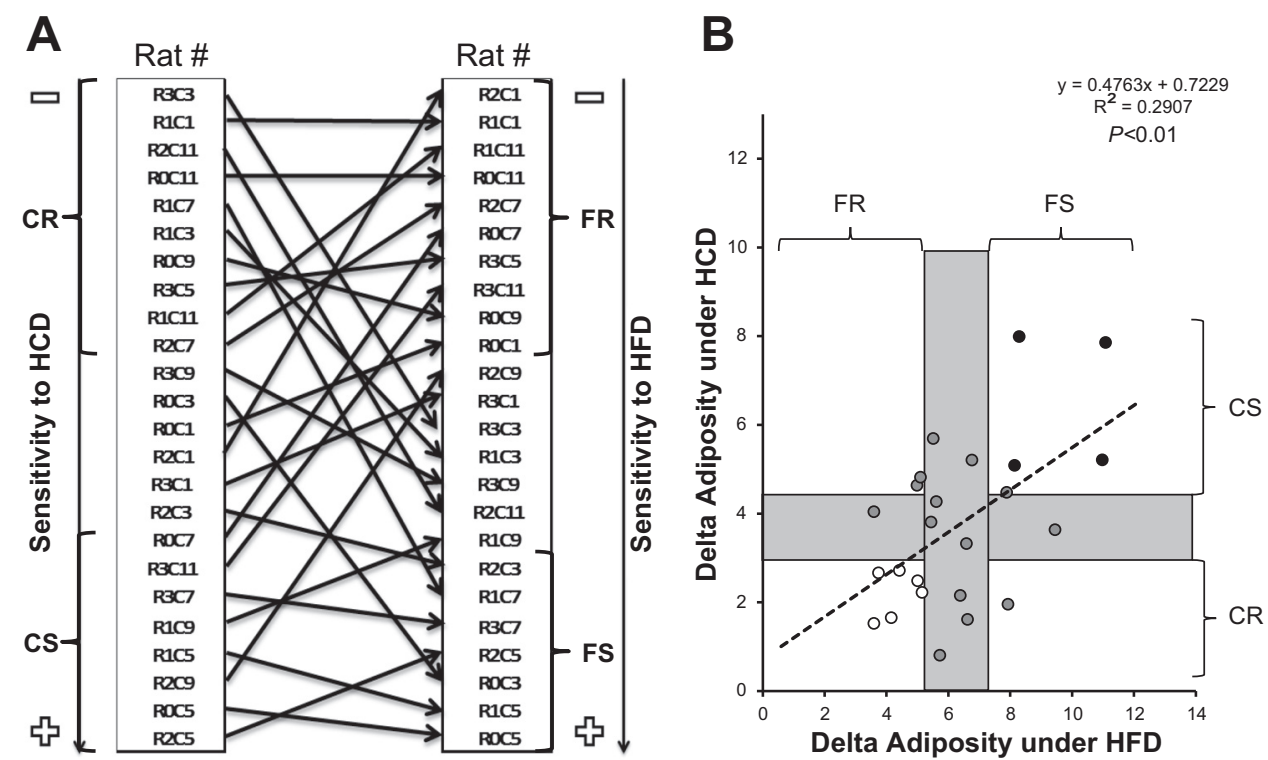

Fig. 1. A: respective sensitivity levels of individuals to high-carbohydrate diet (HCD) and hight-fat diet (HFD). Top to bottom: classification from the lesser to the higher sensitivity to the HCD (left) and HFD (right). Arrows describe the evolution of each individual in the classification. Descending arrows mean less sensitive to HCD and more to HFD, ascending arrows more sensitive to HCD and less to HFD, and horizontal arrows sensitive or resistant to both. B: correlation between adiposity gains under HFD and HCD. Horizontal shaded area limits the intermediate gain of adiposity between HCD sensitive (above) and HCD resistant ones (below). Similarly, vertically shaded area limits the intermediate gain of adiposity between HFD-resistant rats (left) and HFD-sensitive rats (right). Open symbols denote rats resistant to both diets, while black symbols denote rats sensitive to both diets, and gray symbols indicate rats resistant-intermediate to one diet and sensitive-intermediate to the other. Despite significance, the correlation shows that resistance or sensitivity to one diet is poorly predictive of the resistance or sensitivity to the other $\left(R^{2}=0.29\right)$. 

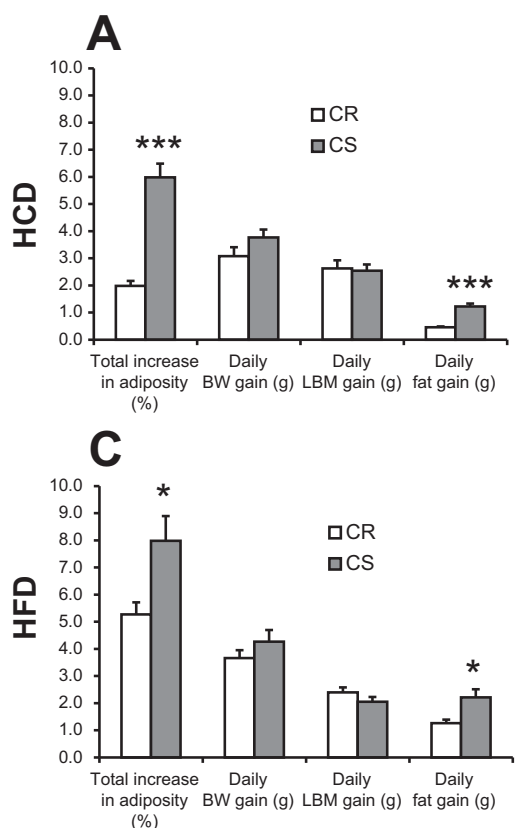
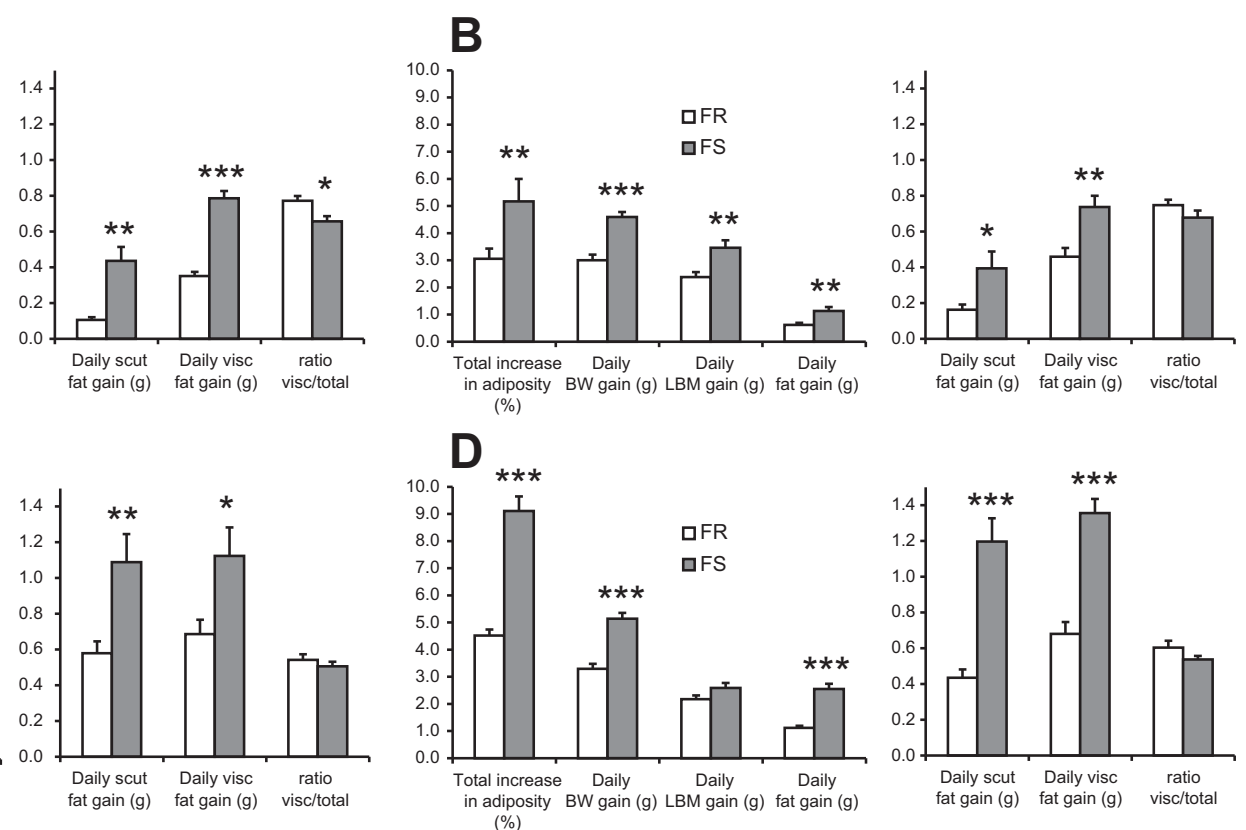

Fig. 2. Evolution of body composition in carbohydrate-resistant-carbohydrate-sensitive (CR-CS) $(A$ and $C$ ) and fat-resistant-fat-sensitive (FR-FS) $(B$ and $D)$ rats. Note that CS rats are characterized by increased adiposity without significant differences in fat-free mass (FFM) and body weight (BW) gain, whereas in FS rats, adiposity, FFM, and $\mathrm{BW}$ are all significantly increased. $* P<0.05$, $* * P<0.01, * * * P<0.001$.

of the high correlation between adiposity gain and BW gain under HFD (Fig. 3), BW can be taken as a proxy of adiposity gain when rats are fed a HFD. This characteristic was used in experiment 2 to remove the necessity of MRI. In contrast, under $\mathrm{HC}$ feeding, BW gain only poorly reveals the differences in adiposity gain between individuals (Fig. 3), and dual photon beam absorptiometry, MRI, or an equivalent noninvasive method, is required to separate CR from CS rats.

Table 3. mRNA expression (arbitrary units) in adipose tissue (expression relative to $18 S$ ) and in the hypothalamus (expressed relative to RPL 13A)

\begin{tabular}{lccc}
\hline \hline & FR $(n=10)$ & FS $(n=7)$ & $P$ \\
\hline & Adipose Tissue $($ Expression Relative to $18 S)$ & \\
ACC & $1.56 \pm 0.171$ & $2.01 \pm 0.653$ & NS \\
FAS & $43.2 \pm 7.17$ & $51.15 \pm 10.7$ & NS \\
Lept & $5.55 \pm 0.624$ & $7.81 \pm 0.950$ & 0.06 \\
LPL & $0.320 \pm 0.0334$ & $0.299 \pm 0.048$ & NS \\
& Hypothalamus $($ Expressed Relative to RPL $13 A)$ & \\
NPY & $3.013 \pm 0.408$ & $3.523 \pm 0.258$ & NS \\
AGRP & $4.810 \pm 0.690$ & $6.662 \pm 0.587$ & 0.08 \\
POMC & $33.026 \pm 5.641$ & $50.540 \pm 4.354$ & 0.04 \\
CART & $1.724 \pm 0.119$ & $2.006 \pm 0.039$ & 0.09 \\
MC4-R & $3.530 \pm 0.235$ & $3.783 \pm 0.242$ & NS \\
INSULIN-R & $10.650 \pm 0.536$ & $11.293 \pm 0.603$ & NS \\
FTO & $13.673 \pm 1.450$ & $15.195 \pm 1.606$ & NS \\
ObR & $8.720 \pm 0.699$ & $7.879 \pm 0.902$ & NS \\
CRF & $17.002 \pm 0.038$ & $16.938 \pm 0.078$ & NS \\
\hline
\end{tabular}

ACC, acetyl-CoA carboxylase; FAS, fatty acid synthase; Lept, leptin; LPL, lipoprotein lipase; NPY, neuropeptide Y; AGRP, Agouti-related peptide; IR, insulin receptor; POMC, proopiomelanocortin; CART, cocaine- and amphetamine-regulated transcript; MC4R, melanocortin-4 receptor; INSULIN-R, insulin receptor; FTO, fat mass and obesity-associated gene; ObR, leptin receptor, long cytoplasmic form; CRF, corticotropin-releasing factor; FR, fat-resistant; FS, fat-sensitive; NS, nonsignificant.

\section{CI, TEE, and EB under HFD (Experiment 1)}

$\mathrm{CR}$ and CS rats did not exhibit any difference in CI or TEE regardless of the procedure used to adjust the data for differences in body size and composition, nor differences in the structure of feeding [meal size, meal number, speed of ingestion, day/night distribution (data not shown)] nor in the pattern of spontaneous activity (Fig. 4, top). In FS rats, caloric intake was $25 \%$ larger $(P=0.02)$ than in FR rats, but adjustment to FFM or to AMM reduced this difference to $15 \%(P=0.03)$ and $13 \%$, respectively $(P=0.06)$ (Fig. 4 , bottom). TEE was $20 \%$ larger in FS rats, but adjustment to FFM or AMM reduced the difference to $10 \%$ (NS) and 8\% (NS), respectively (Fig. 4, bottom). Taken

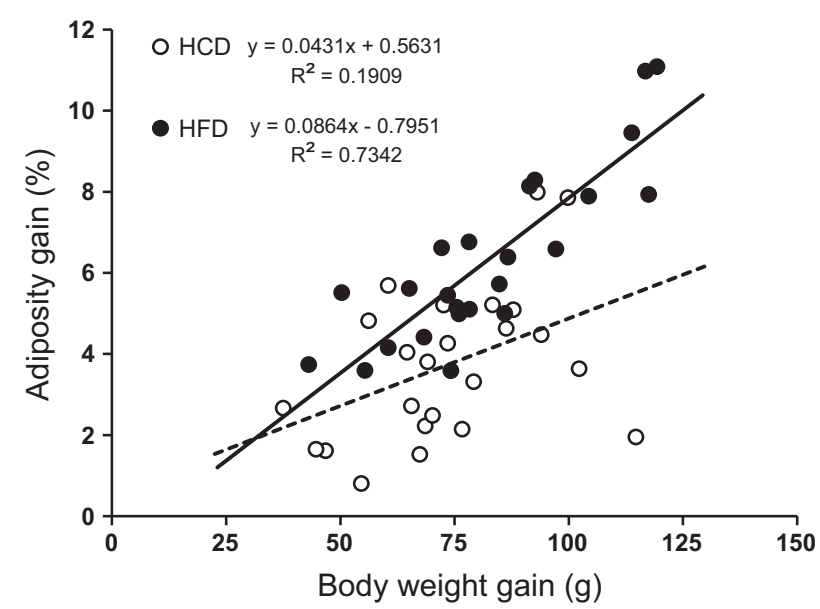

Fig. 3. Correlation between BW gain and adiposity gain under HCD and HFD. Adiposity gain is highly correlated to BW gain under HFD, which permits us to use BW gain as a proxy for the sensitivity to HFD. In contrast, under a HCD, adiposity gain is poorly correlated to BW gain, and analysis of body composition is required to discriminate between sensitive and resistant rats. 


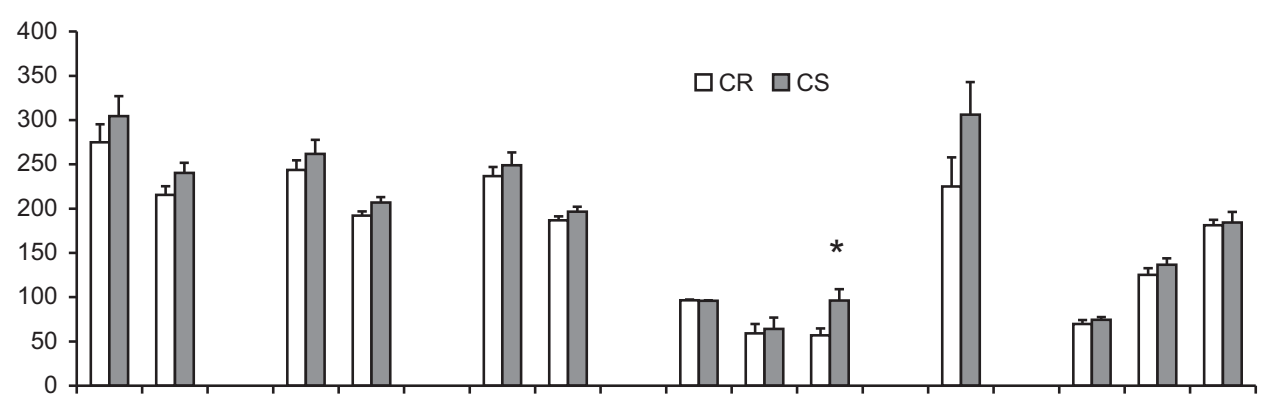

Fig. 4. Components of energy balance in $\mathrm{CR}$ $(n=7)$ and CS $(n=6)($ top $)$ and FR $(n=$ $7)$ and FS $(n=5)$ rats (bottom) after adaptation to the HFD. During the $3 \mathrm{wk}$ on a HFD, CS rats did not differ from CR rats on any components of energy expenditure, but energy balance (EB) during the 21 days of a $\mathrm{HCD}$, estimated from changes in body composition, was larger in CS rats. FS rats, in contrast, exhibited a higher caloric intake (CI) and energy expenditure (EE) on a per rat basis but not after adjustment to active metabolic mass $(\mathrm{AMM}=\mathrm{FFM}+0.2 \times$ FM), where FFM is fat-free mass and FM is fat mass. EB was also significantly higher during the 21 days of HFD, as well as during the 5 days during which components of $\mathrm{EE}$ were measured. Mean intensity of activity was the same as in FR rats, but was dispatched differently, FS rats spending more time active but exhibiting significantly lower intensity of activity bouts. $* P<0.05, * * P<$ $0.01, * * * P<0.001$ (AU, arbitrary units).

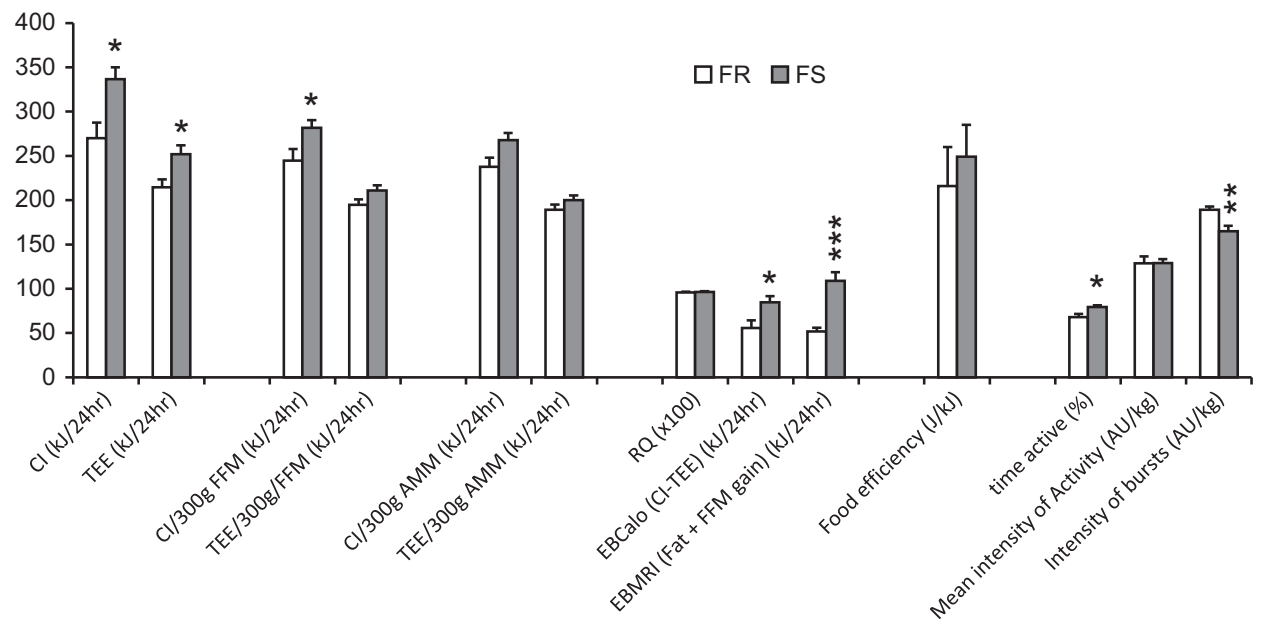

together, these observations show that FS rats tend to eat significantly more but have a level of energy expenditure not significantly higher than that of FR rats, or, said another way, in FS rats, the increase in EE $(8-10 \%)$ under HF feeding did not fully compensate for the increase in CI (13-15\%), which explains the more positive energy balance of these rats.

EB computed from EBcalo and EBMRI (see MATERIALs AND METHODS) produced the same values in CR and FR rats, but EB computed from EBMRI resulted in larger values than EBCalo in CS and, in particular, in FS rats (Fig. 4). Detailed analysis of body weight gain during the HFD period showed that FS rats indeed gained slightly less BW during the 4 days in the metabolic cages than during the overall $21 \mathrm{~d}$ of HFD (5.48 vs. $4.06 \mathrm{~g} / \mathrm{d}, P=0.13$ ), while FR ones continued to gain weight identically $(3.70 \mathrm{~g} /$ day vs. $3.63 \mathrm{~g} /$ day, $P=$ 0.92). It can, thus, be inferred that FS rats (and to a lesser extent CS rats) ate less while in the metabolic cages than in their home cages while their respective FR and CR partners maintained a constant intake. Nevertheless, these results show that, under HFD, FS rats gained more fat and weight because they increased CI more than TEE. The difference being small, food efficiency did not appear significantly increased in FS rats and no differences in RQ were observed with FR ones (Fig. 4, bottom).

Considering spontaneous activity, FS rats spent significantly more time active (Fig. 4, bottom). However, mean daily work due to activity was similar to that in FR ones because when active, FS rats developed bursts of activity of significantly lower intensity than FR rats (Fig. 4, bottom). In addition, significant inverse relationships were found between the intensity of the burst of activity and body fat gain $\left(r^{2}=0.39, n=12 ; P<0.02\right)$, adiposity gain $\left(r^{2}=0.39, n\right.$ $=12 ; P<0.02)$ and energy balance computed from changes in body composition measured by MRI (EBMRI) $\left(r^{2}=0.40\right.$, $n=12 ; P<0.02)$.

\section{Detailed Analysis of the Components of Energy Expenditure in FR/FS Rats under HFD (Experiment 2)}

On the basis of the results of experiment 1 (Fig. 3), in experiment 2, FR and FS rats were segregated according to BW gain. Accordingly, FS rats gained $5.88 \pm 0.36 \mathrm{~g} / \mathrm{day}$ and FR ones $3.22 \pm 0.36 \mathrm{~g} /$ day $(P<0.0001)$ during HFD, and their mean weights at the end of the HFD period were $395.1 \pm 10.3 \mathrm{~g}$ vs. $326.4 \pm 8.7$, respectively $(P<0.001)$. Analysis of body composition by MRI performed at the end of the HFD period also indicated that FS rats had significantly more body fat $(52.66 \pm 3.55$ vs $37.39 \pm 3.32 \mathrm{~g}, P<$ 0.01 ).

The time-course changes in RMR and RQ measured on the FR and FS rats from $10 \mathrm{~h}$ before to $6 \mathrm{~h}$ after ingestion of the 60-kJ HFD test meal are shown in Fig. 5. RMR was significantly higher in FS rats (Fig. 5A), but only as a result of their larger body size as an adjustment to FFM (Fig. $5 B$ ) and to AMM (Fig. 5C) completely erased the difference. Relative to meal time $(0 \mathrm{~h})$, premeal ( -10 to $0 \mathrm{~h})$ and postmeal $(0$ to $6 \mathrm{~h}$ ), RQs were also similar in the two groups (Fig. 5D), indicating that the ratio of lipid to glucose oxidation was similar in FR and FS rats in the postabsorptive, as well as in the fed state. The other components of energy expenditure (Fig. 6) also showed no differences between FR and FS rats. Taken together with the data of experiment 1 , these results suggest that the FS 

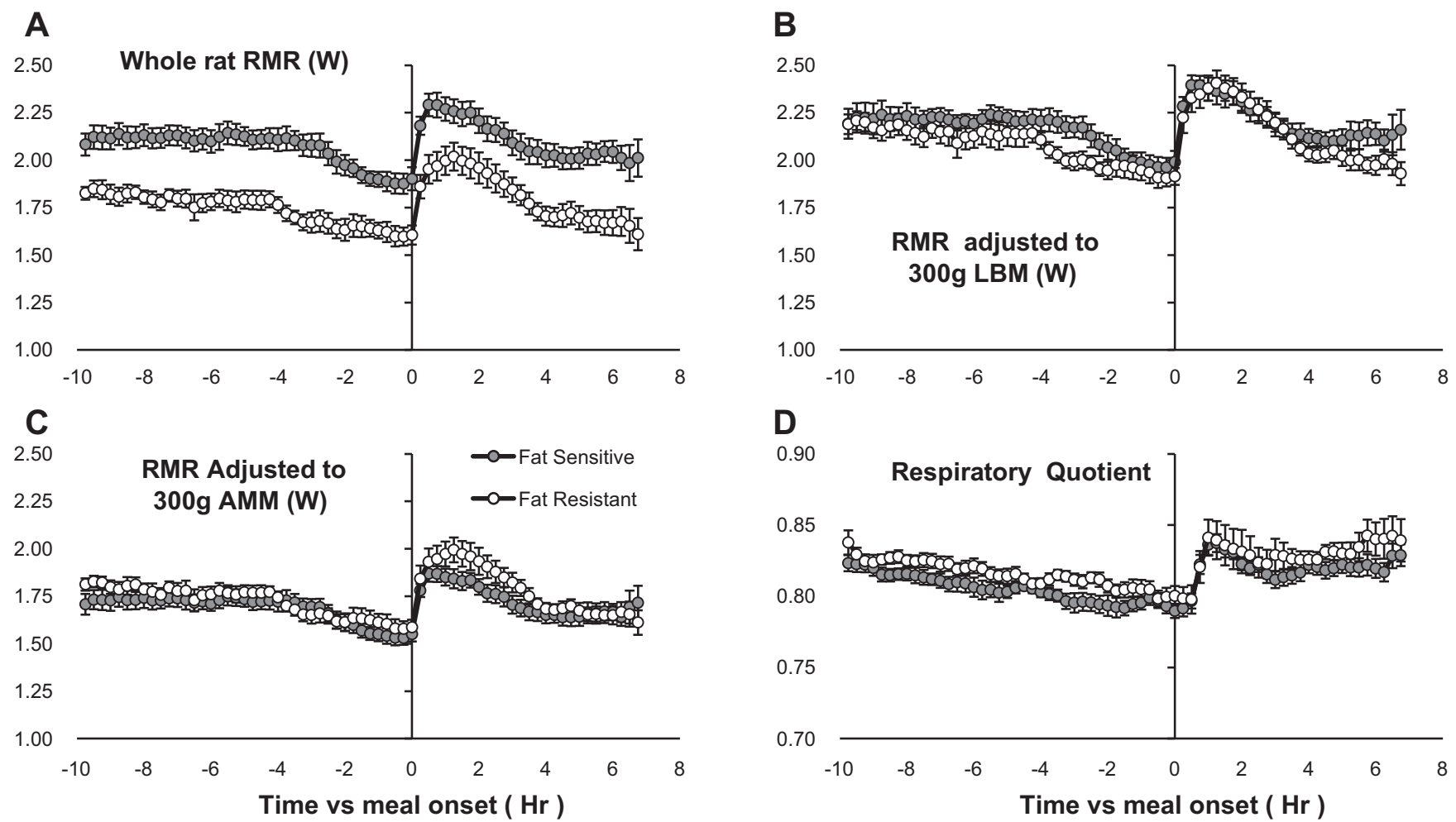

Fig. 5. Evolution (means $\pm \mathrm{SE}$ ) of resting metabolic rate (RMR) ( $A$ : whole rat, $B$ : adjusted to FFM, $C$ : adjusted to AMM) and respiratory quotient (RQ) in the fasted state $(-10$ to $0 \mathrm{~h})$ and in response to ingestion of the 60-kJ test meal of the HFD $(0$ to $6 \mathrm{~h})$ in FR $(n=10)$ and FS $(n=7)$ rats. $A$ : whole rat RMR was larger in FS rats, but adjustment of RMR to LBM or AMM ( $B$ and $C$ ) shows that this was only the result of the larger body size of FS rats. In $A$ and $D$, it is also apparent that postingestive RQ, thermic effect of feeding (TEF), and meal-induced changes in RQ are similar in FR and FS rats (see also Fig. 6) [Note that TEF must not be estimated from $B$ and $C$ because adjustment to body size overestimates meal-induced changes in RMR in smaller rats (here FR ones)].

rats did not suffer any defect in the various components of energy expenditure.

Blood Parameters and mRNA Expression in the Liver, Adipose Tissue, and the Hypothalamus in FR and FS Rats after HFD (Experiment 1)

Plasma TG was higher in FS than in FR rats $(2.61 \pm 0.12$ vs. $2.06 \pm 0.12, P<0.01)$. No differences were observed for ketone bodies, free fatty acids, total cholesterol, or HDL cholesterol (results not shown). TG in the liver $(4.99 \% \pm$ 0.52 in FS vs. $4.13 \% \pm 0.33$ in FR) and epididymal adipose tissue $(92.1 \% \pm 2.3$ in FS vs. $89.8 \pm 2.7$ in FR) were not different in FR and FS rats. These results indicate that after 3 wk of HFD, the FS rats did not yet suffer complications due to obesity.

No differences in gene expression of enzymes involved in glucose and lipid metabolism were observed between groups in the liver (data not shown). In adipose tissue, only
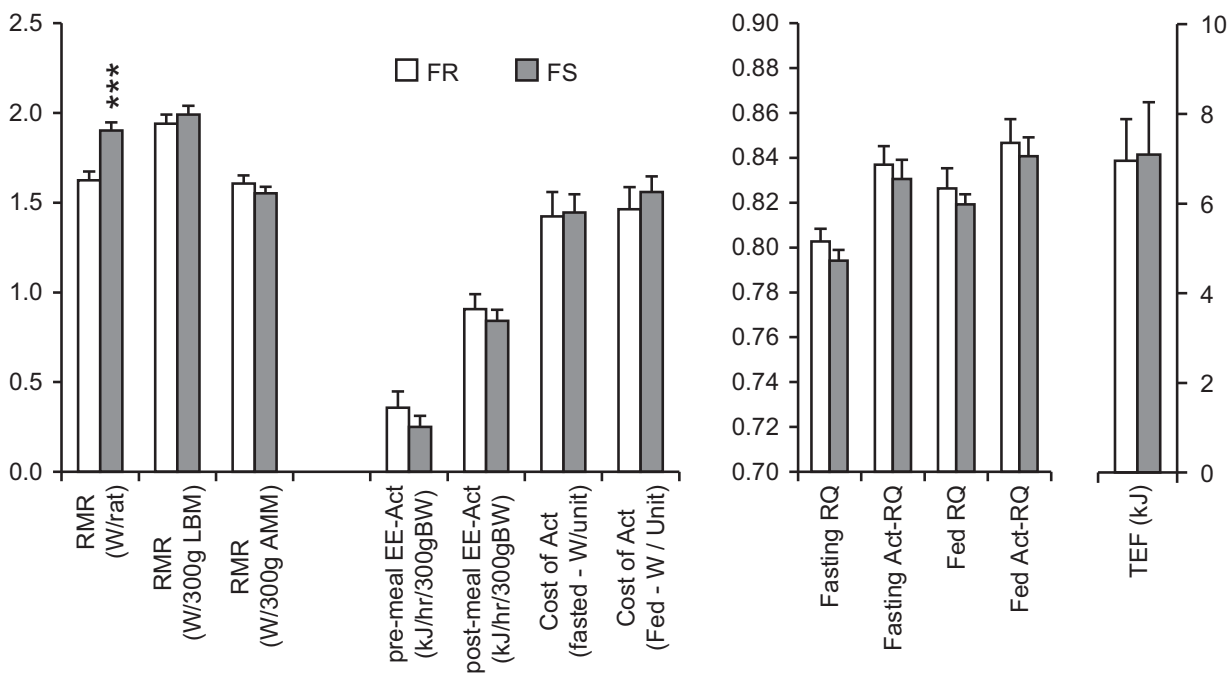

Fig. 6. Components of energy expenditure in FR $(n=10)$ and FS $(n=7)$ rats during a cycle of fasting and refeeding under HFD showed that only whole rat RMR was significantly larger in FS rats but exclusively because of the larger LBM and MMA of FS rats. Indeed, if adjusted to body size, none of the components of energy expenditure differed between FR and FS rats, indicating that in this model, the predisposition to obesity under HFD did not result from any metabolic defect at the level of RMR, TEF, substrate oxidation, EE with activity, and cost of activity. $* * * P<0.001$. 
leptin mRNA tended to be greater in FS rats $(P=0.056)$ (Table 3), which was in accordance with their higher adiposity. In the hypothalamus, POMC was higher and Agouti-related peptide (AGRP) and cocaine- and amphetamine-regulated transcript (CART) tended to be higher as well (Table 3).

\section{DISCUSSION}

The present study confirms that significant differences in body adiposity can develop during HFD but also during HCD feeding, and reveals that sensitivity or resistance to adiposity gain under HCD only partly covers the population of rats sensitive or resistant to HFD. These results together with analysis of body composition, components of energy expenditure, spontaneous activity, and metabolic markers indicate that the sensitivities to a HFD or a HCD have different origins and that FS rats do not exhibit any defect in any component of energy expenditure.

Although adiposity gain is lower in absolute terms in CS rats under HCD than in FS rats under HFD, the difference in adiposity gain is larger between $\mathrm{CR}$ and $\mathrm{CS}$ rats under HCD than between FR and FS rats under HFD. This confirms previous observations that individuals can be classified not only by their HFD sensitivity (classical obesity-prone) but also by their HCD sensitivity $(15,34)$. This is observed in conditions in which the HCD was based on the AIN93 recommendation, in which most of the carbohydrate is provided as starch (38) in contrast to those diets designed to induce overfeeding and/or insulin resistance through the introduction of a high proportion of sucrose, as in the few previous studies that reported differences in sensitivity to HCD (10). Indeed, we observed a significant correlation between adiposity gain under HCD and HFD, but as testified by the rather low $R^{2}$ value, the level of adiposity gain under HFD explains only $30 \%$ of the adiposity gain from the HFD, suggesting that only some of the mechanisms underlying sensitivity to HCD are involved in the sensitivity to HFD and vice versa. An important observation was that in CS rats on a $\mathrm{HCD}$, dietary fat accumulates significantly more viscerally than in FS rats on a HFD, which in the long term may be an aggravating factor for CS rats, considering that visceral fat is more deleterious than the subcutaneous sort $(6,9)$. However, we also observed that both CS and FS rats increased subcutaneous fat deposition more than visceral fat deposition and that in absolute terms, CS rats finally deposited $\sim 2$ times less visceral fat than FS rats. Thus, long-term studies are required to estimate the evolution of pathologies in CS rats. It is poorly probable that these differences were the result of the fact that the rats were first submitted to HCD because, in practice, in most studies before being submitted to a HFD, rats are fed on chow, i.e., on a $\mathrm{HCD}$. Also, $\mathrm{BW}$ and $\mathrm{BW}$ gain were higher during HFD than during HCD; therefore, the larger visceral fat deposition under HCD cannot be the result of a higher growth rate during the HCD. Differences in adiposity gain were also recently observed in C57BL/6 mice fed a chow diet (meaning a HCD close in composition to the one used here) associated with differential expression of proteins involved in energy metabolism, glycolysis, and fat synthesis in visceral adipose tissue (51). This suggests that 1) preferential visceral fat accumulation may be a common trait of high-carbohydrate diets, 2) sensitivity to HCD is probably a common feature of various species, and 3) metabolic pathways orienting fat depo- sition viscerally rather than subcutaneously are involved. The fact that liver TG was highest in CS rats suggests that lipid synthesis in the liver may be the source of this specific distribution. In clinical practice, this may be a parameter to consider before dietary interventions, because in subjects with a high visceral to subcutaneous fat ratio, low-fat diets may not necessarily be advisable.

Comparing TEE and CI and considering the consistent results provided after adjustment to FFM only or AMM (FFM + $0.2 \times \mathrm{FM})(2,14)$ indicates that after adaptation to HFD, there were no differences in TEE or CI between CR and CS rats, confirming their overall low sensitivity to HFD, whereas FS rats ate more and had a larger TEE than FR ones. On the other hand, these data also show that in FS rats TEE is not decreased, but increased less than $\mathrm{CI}$, which explains their more positive EB. Therefore, FS rats do not gain more fat because of a reduced TEE during HFD or, since RQ was also not different, because of a reduced rate of lipid oxidation, but because they ate more. This result is confirmed by the detailed analysis of the components of energy expenditure, which did not reveal any defects. FS rats, indeed, exhibited increased RMR, but this increase completely disappeared after adjustment for differences in body composition. RQ, reflecting the relative rates of glucose and lipid oxidation, was the same in the fasting state, as well as in response to ingestion of the test meal, indicating that the capacity of the FS rats to adjust glucose and lipid oxidation to overnight food restriction, as well as to respond to ingestion of a high-fat test-meal, was the same as in FR rats. This was also true for fuel use by the working muscles, as testified by the same activity (Act)-RQ and the same cost of activity in FR and FS rats. The present data, together with previous studies $(15,34)$, thus, strongly suggest that there is no defective regulation of energy metabolism in FS rats. This is not true for CS rats, for which we previously reported exaggerated changes in glucose and lipid oxidation in response to ingestion of a high-carbohydrate test meal (34).

On the energy intake side of the energy balance equation, many arguments are in favor of a defective control of food intake in FS rats. HFDs are generally less satiating than HCDs $(33,49)$, and FS rats are suspected to be less sensitive to the satiating effect of fat (12) or to be more responsive to the palatability of HFDs, which seems to be the case here. In addition, the fact that FS rats, but not FR ones, reduced their caloric intake when housed in the calorimetry cages suggests that they had less motivation for feeding than the FR ones. Such lower motivation to eat was also reported in $o b / o b$ mice following operant training (39) and by Shin et al. in 2011 (42), who also reported that high-fat diet-induced obesity paradoxically decreases motivation for food reward, as measured by incentive runway and progressive ratio lever press performance. They concluded, and the present results are in line with this, that while obese rats readily indulge in easily available palatable fatty food, they are not ready to make an extra effort to obtain it.

Only a few studies have investigated the expression of hypothalamic neuropeptides in FS or FR animals, with inconsistent results. Resistance to the central anorectic effect of leptin and insulin seems to be present, even before the onset of obesity (27-29). In this study, we observed no difference in the mRNA expression of insulin and leptin receptors, but this does not preclude the fact that entry of leptin into the brain may be 
impaired, in particular, following the observed increase in plasma TG (see below). POMC and AGRP expression in the arcuate nucleus is lower in diet-induced obese (DIO) mice than in diet-resistant (DR) mice after 22 wk of HFD (21), but no difference was observed in arcuate NPY or POMC mRNA between DIO and DR rats after 4 wk on a high-energy diet (46). Here, we show that hypothalamic AGRP mRNA expression tended to be larger in FS rats $(P=0.06)$, but that of POMC, which exerts an anorectic effect, was also increased $(P<0.04)$. The polymorphism of outbred FS (and probably CS) rats probably explains the difficulty of describing a clear brain profile. On the other hand, although many studies have demonstrated that the level of mRNA expression can be used as an indicator of functional significance of the neurotransmitter system, confirmation is still needed at the protein and functional levels.

FS rats have been characterized as "high plasma TG responders" $(11,23)$. In our hands, FS rats on a HFD (but not on a HCD, data not shown) had increased plasma TG, which agrees with the results of many previous studies and the hypothesis that increased TG levels in response to high-fat feeding is a factor predicting and perhaps predisposing rats for sensitivity to a HFD. One suggested mechanism is that TG impairs leptin signaling in the brain (4), which would stimulate feeding. Reduced whole body $(8,41)$ and liver FFA oxidation (22) in response to a high-fat diet have also been quoted as predisposing factors. In this study, however, plasma FFA and ketone bodies are not affected and whole body RQ measured under free feeding conditions was the same in FR and FS rats, which indicates that after 2-3 wk of adaptation and despite a still higher rate of weight gain, there was no difference in the ratio of glucose to lipid oxidation at the whole body level. This result is in line with a previous report from our laboratory that after introduction of a HFD, adjustment of whole body fat oxidation to the higher fat content of the diet (as measured from the speed of adjustment of whole body RQ to the food quotient) tended to be slower in FS rats but was adjusted within 4-5 days (15). The present results also agree with a report by Novak et al. (35), who observed that in response to high-fat feeding, RQ decreased to the same extent in OP and OR rats (35). In addition, we also reported that the RQ and TEF responses to a HFD test meal were not different between the FR and FS rats of this study during the 3 wk they were maintained on the HCD (34), and we show here that after 3 wk of HFD, there is still no difference in the metabolic responses to ingestion of a HF test meal between FR and FS rats. Thus, our results suggest that a defective lipid oxidation may participate in the rapid rate of weight gain of FS rats during the first few days of adaptation to HFD (dynamic phase), but not to the sustained increase in BW gain observed over the long term. It is, however, disturbing that RQ was not higher in FS rats because if FS rats stored more fat than FR ones, they should have oxidized a lower proportion of the dietary fat, and the RQ should necessarily be higher (32). One possibility to explain this apparent discrepancy is that in FS rats, more energy may be derived from polysaccharide catabolism by the microbiota, thus delivering short-chain fatty acids into the circulation (47). Indeed, studies of germ-free and conventionalized mice revealed that the microbiota promote absorption of monosaccharides from the gut lumen, with a resulting induction of de novo hepatic lipogenesis, and that $o b / o b$ mice are able to extract more energy from ingested food substances than their lean littermates $(3,47)$.

A decreased level of physical activity in obesity-prone mice fed a HFD has previously been reported $(5,35)$, but this does not definitely preclude less energy being expended with activity because activity is usually measured with beam breaks, which does not take into account the fact that the cost of moving is higher in heavier OP rats or mice. For example, Novak et al. (35) reported a decreased overall activity measured by beam breaks in diet-induced obese rats $(\sim 28$ vs. 34 estimated from their Fig. 1 in Ref. 35), but the obese rats were much heavier than the lean ones ( $352 \mathrm{~g}$ vs. $250 \mathrm{~g}$ ). Thus, the energy expended with activity as computed by activity $X$ weight $(\mathrm{kg})$ was, in fact, higher in OP than in OR rats (18.9 vs. 15.95). What Novak et al. (35) also observed was rather small differences in horizontal activity but striking differences in vertical activity. Considering this point with the fact that we observed that the intensity of the bursts of activity but not overall activity was different between FR and FS rats, it seems that the pattern of activity and the types of activity in which the FR and FS rats engage rather than overall activity may be different and contribute to the sensitivity or resistance to diet-induced obesity. An interesting point is that in this study, we observed an inverse correlation between intensity of the bursts of activity and adiposity gain, fat gain, and energy balance, and in a study that we published in 2011, we observed a strong inverse correlation between the sensitivity of CR and CS rats to HCD and the intensity of the bursts of activity under HCD $\left(r^{2}=0.76\right)$ and furthermore, that this correlation disappeared within days of the rats being switched to the HFD (15). That bursts of activity are of smaller intensity in CS rats only under HCD and in FS rats only under HFD suggests that spontaneous activity can be a component involved in the predisposition to body fat gain, whatever the fat content of the diet. Differences in muscle fiber types have been reported between FS and FR rats (1) and lean and obese subjects (48). Whether such differences can affect the stereotypic behavior of the rats and/or the control of energy metabolism in working muscles and affect energy balance remains to be elucidated. Wade et al. (48), for example, reported that fatter men with a low proportion of slow muscle fibers combusted less fat during cycle ergometry. In our hands, however, we did not observe such a defect in fat oxidation in obese Zucker rats running on a treadmill (40) despite the fact that it was reported that obese Zucker rats have a greater proportion of fast-twitch fibers (19), and in the present, as well as in our previous study (34), no differences in Act-RQ and in the energetic cost of activity were observed, suggesting that the reduced intensity of activity bursts is not accompanied by clear defects in the metabolism of the working muscles. However, we still did not perform a detailed analysis of the short-term changes in energy metabolism induced by the bursts of activity. Such specific studies must be designed to further analyze if differences in the intensity of bursts can affect substrate oxidation in muscles and energy balance.

In conclusion, the present data together with that which we previously published suggest that the propensity to gain weight on a HFD in FS rats is not necessarily sustained by a low metabolic rate, a low energy expended with activity, a low cost of activity or a poor capacity to oxidize fat, but only by an excess CI $(\sim 15 \%)$ not fully balanced by a compensatory 
increase in EE $(\sim 10 \%)$. High blood TG levels appear to be a constant in FS rats fed a HFD and can possibly stimulate feeding by impairing leptin signaling to the brain. A common point of CS and FS rats is an altered pattern of spontaneous activity, characterized by a reduced intensity of the bursts of activity when the rats are fed the diet to which they are sensitive.

\section{Perspectives and Significance}

Significant increases in adiposity can develop under a HCD in rats that can otherwise be sensitive or resistant to HFDinduced obesity, and the data we obtained hitherto suggest that different metabolic flaws may operate. At a phenotypic level, CS rats on a HCD, contrary to FS rats under HFD, do not enlarge their LBM, and fix more fat viscerally, and thus are more difficult to identify. Since carbohydrate sensitivity has also been reported in mice, it is plausible that this characteristic may be present in humans. Obviously, the nutritional treatment to apply to carbohydrate- and fat-sensitive individuals must be different. In our hands, FS rats seem to be particularly sensitive to the reward signals of palatable high-fat food, suggesting that future studies should focus on the reward signaling in these animals. In contrast, CS rats exhibit a defective substrate partitioning in response to ingestion of a high-carbohydrate meal, and future studies should focus on glucose responsiveness, insulin sensitivity, and liver metabolism to clarify the underlying mechanisms.

\section{DISCLOSURES}

No conflicts of interest, financial or otherwise, are declared by the authors.

\section{AUTHOR CONTRIBUTIONS}

Author contributions: D.A.-M., C.C., and P.C.E. conception and design of research; D.A.-M., C.C., J.P., and P.C.E. performed experiments; D.A.-M., C.C., N.A.N., J.P., and P.C.E. analyzed data; D.A.-M., N.A.N., D.T., and P.C.E. interpreted results of experiments; D.A.-M., C.C., N.A.N., G.F., D.T., and P.C.E. edited and revised manuscript; D.A.-M., C.C., N.A.N., J.P., G.F., D.T., and P.C.E. approved final version of manuscript; D.T. and P.C.E. drafted manuscript; P.C.E. prepared figures.

\section{REFERENCES}

1. Abou Mrad J, Yakubu F, Lin D, Peters JC, Atkinson JB, Hill JO. Skeletal muscle composition in dietary obesity-susceptible and dietary obesity-resistant rats. Am J Physiol Regul Integr Comp Physiol 262: R684-R688, 1992.

2. Arch JR, Hislop D, Wang SJ, Speakman JR. Some mathematical and technical issues in the measurement and interpretation of open-circuit indirect calorimetry in small animals. Int J Obes 30: 1322-1331, 2006.

3. Backhed F, Ding H, Wang T, Hooper LV, Koh GY, Nagy A, Semenkovich CF, Gordon JI. The gut microbiota as an environmental factor that regulates fat storage. Proc Natl Acad Sci USA 101: 15718-15723, 2004.

4. Banks WA, Coon AB, Robinson SM, Moinuddin A, Shultz JM, Nakaoke R, Morley JE. Triglycerides induce leptin resistance at the blood-brain barrier. Diabetes 53: 1253-1260, 2004.

5. Bjursell M, Gerdin AK, Lelliott CJ, Egecioglu E, Elmgren A, Tornell J, Oscarsson J, Bohlooly YM. Acutely reduced locomotor activity is a major contributor to Western diet-induced obesity in mice. Am J Physiol Endocrinol Metab 294: E251-E260, 2008.

6. Catalano KJ, Bergman RN, Ader M. Increased susceptibility to insulin resistance associated with abdominal obesity in aging rats. Obes Res 13: 11-20, 2005.

7. Chang GQ, Karatayev O, Barson JR, Chang SY, Leibowitz SF. Increased enkephalin in brain of rats prone to overconsuming a fat-rich diet. Physiol Behav 101: 360-369, 2010.
8. Chang S, Graham B, Yakubu F, Lin D, Peters JC, Hill JO. Metabolic differences between obesity-prone and obesity-resistant rats. Am J Physiol Regul Integr Comp Physiol 259: R1103-R1110, 1990.

9. Despres JP. Excess visceral adipose tissue/ectopic fat the missing link in the obesity paradox? J Am Coll Cardiol 57: 1887-1889, 2011.

10. Dourmashkin JT, Chang GQ, Gayles EC, Hill JO, Fried SK, Julien C, Leibowitz SF. Different forms of obesity as a function of diet composition. Int J Obes 29: 1368-1378, 2005.

11. Dourmashkin JT, Chang GQ, Hill JO, Gayles EC, Fried SK, Leibowitz SF. Model for predicting and phenotyping at normal weight the long-term propensity for obesity in Sprague-Dawley rats. Physiol Behav 87: 666-678, 2006.

12. Duca FA, Sakar Y, Covasa M. Combination of obesity and high-fat feeding diminishes sensitivity to GLP-1R agonist exendin-4. Diabetes 62 : 2410-2415, 2013.

13. Even PC, Mokhtarian A, Pele A. Practical aspects of indirect calorimetry in laboratory animals. Neurosci Biobehav Rev 18: 435-447, 1994.

14. Even PC, Nadkarni NA. Indirect calorimetry in laboratory mice and rats: principles, practical considerations, interpretation and perspectives. Am J Physiol Regul Integr Comp Physiol 303: R459-R476, 2012.

15. Even PC, Nadkarni NA, Chaumontet C, Azzout-Marniche D, Fromentin G, Tome D. Identification of behavioral and metabolic factors predicting adiposity sensitivity to both high-fat and high-carbohydrate diets in rats. Front Physiol 2: 96, 2011.

16. Even PC, Rolland V, Roseau S, Bouthegourd JC, Tome D. Prediction of basal metabolism from organ size in the rat: relationship to strain, feeding, age, and obesity. Am J Physiol Regul Integr Comp Physiol 280: R1887-R1896, 2001.

17. Ferrannini E. The theoretical bases of indirect calorimetry: a review. Metabolism 37: 287-301, 1988.

18. Fink BD, Herlein JA, Almind K, Cinti S, Kahn CR, Sivitz WI. Mitochondrial proton leak in obesity-resistant and obesity-prone mice. Am J Physiol Regul Integr Comp Physiol 293: R1773-R1780, 2007.

19. He D, Bolstad G, Brubakk A, Medbo JI. Muscle fibre type and dimension in genetically obese and lean Zucker rats. Acta Physiol Scand 155: 1-7, 1995.

20. Hill JO, Peters JC. Environmental contributions to the obesity epidemic. Science 280: 1371-1374, 1998.

21. Huang XF, Han M, South T, Storlien L. Altered levels of POMC, AgRP and MC4-R mRNA expression in the hypothalamus and other parts of the limbic system of mice prone or resistant to chronic high-energy dietinduced obesity. Brain Res 992: 9-19, 2003.

22. Ji H, Friedman MI. Reduced hepatocyte fatty acid oxidation in outbred rats prescreened for susceptibility to diet-induced obesity. Int J Obes 32: 1331-1334, 2008.

23. Karatayev O, Gaysinskaya V, Chang GQ, Leibowitz SF. Circulating triglycerides after a high-fat meal: predictor of increased caloric intake, orexigenic peptide expression, and dietary obesity. Brain Res 1298: 111-122, 2009.

24. Lauterio TJ, Barkan A, DeAngelo M, DeMott-Friberg R, Ramirez R. Plasma growth hormone secretion is impaired in obesity-prone rats before onset of diet-induced obesity. Am J Physiol Endocrinol Metab 275: E6-E11, 1998.

25. Levin BE. Reduced norepinephrine turnover in organs and brains of obesity-prone rats. Am J Physiol Regul Integr Comp Physiol 268: R389R394, 1995.

26. Levin BE and Dunn-Meynell AA. Dysregulation of arcuate nucleus preproneuropeptide Y mRNA in diet-induced obese rats. Am J Physiol Regul Integr Comp Physiol 272: R1365-R1370, 1997.

27. Levin BE, Dunn-Meynell AA. Reduced central leptin sensitivity in rats with diet-induced obesity. Am J Physiol Regul Integr Comp Physiol 283: R941-R948, 2002.

28. Levin BE, Dunn-Meynell AA, Banks WA. Obesity-prone rats have normal blood-brain barrier transport but defective central leptin signaling before obesity onset. Am J Physiol Regul Integr Comp Physiol 286: R143-R150, 2004.

29. Levin BE, Dunn-Meynell AA, Ricci MR, Cummings DE. Abnormalities of leptin and ghrelin regulation in obesity-prone juvenile rats. Am J Physiol Endocrinol Metab 285: E949-E957, 2003.

30. Levin BE, Sullivan AC. Glucose-induced sympathetic activation in obesity-prone and -resistant rats. Int J Obes 13: 235-246, 1989.

31. Levy JR, Lesko J, Krieg RJ Jr, Adler RA, Stevens W. Leptin responses to glucose infusions in obesity-prone rats. Am J Physiol Endocrinol Metab 279: E1088-E1096, 2000. 
32. Livesey G, Elia M. Estimation of energy expenditure, net carbohydrate utilization, and net fat oxidation and synthesis by indirect calorimetry: evaluation of errors with special reference to the detailed composition of fuels. Am J Clin Nutr 47: 608-628, 1988.

33. Lucas F, Ackroff K, Sclafani A. High-fat diet preference and overeating mediated by postingestive factors in rats. Am J Physiol Regul Integr Comp Physiol 275: R1511-R1522, 1998.

34. Nadkarni NA, Chaumontet C, Azzout-Marniche D, Piedcoq J, Fromentin G, Tome D, Even PC. The carbohydrate sensitive rat as a model of obesity. PloS One 8: e68436, 2013.

35. Novak CM, Kotz CM, Levine JA. Central orexin sensitivity, physical activity, and obesity in diet-induced obese and diet-resistant rats. Am J Physiol Endocrinol Metab 290: E396-E403, 2006.

36. Pagliassotti MJ, Knobel SM, Shahrokhi KA, Manzo AM, Hill JO. Time course of adaptation to a high-fat diet in obesity-resistant and obesity-prone rats. Am J Physiol Regul Integr Comp Physiol 267: R659R664, 1994.

37. Rada P, Bocarsly ME, Barson JR, Hoebel BG, Leibowitz SF. Reduced accumbens dopamine in Sprague-Dawley rats prone to overeating a fat-rich diet. Physiol Behav 101: 394-400, 2010.

38. Reeves PG, Nielsen FH, Fahey GC Jr. AIN-93 purified diets for laboratory rodents: final report of the American Institute of Nutrition ad hoc writing committee on the reformulation of the AIN-76A rodent diet. J Nutr 123: 1939-1951, 1993.

39. Reynolds MA, Goodrick CL, Ingram DK. Decreased resistance to extinction in ob/ob mice following operant training. Physiol Behav 29: 643-647, 1982.

40. Rolland V, Roseau S, Fromentin G, Nicolaidis S, Tome D, Even PC. Body weight, body composition, and energy metabolism in lean and obese Zucker rats fed soybean oil or butter. Am J Clin Nutr 75: 21-30, 2002 .
41. Schutz Y, Flatt JP, Jequier E. Failure of dietary fat intake to promote fat oxidation: a factor favoring the development of obesity. Am J Clin Nutr 50: 307-314, 1989.

42. Shin AC, Townsend RL, Patterson LM, Berthoud HR. "Liking" and "wanting" of sweet and oily food stimuli as affected by high-fat dietinduced obesity, weight loss, leptin, and genetic predisposition. Am J Physiol Regul Integr Comp Physiol 301: R1267-R1280, 2011.

43. Southgate DAT. Meat, fish, eggs and novel protein. In: Human Nutrition and Dietetics (9th ed.). London: Churchill Livingstone 1993, p. 246.

44. Stepien M, Gaudichon C, Fromentin G, Even P, Tome D, AzzoutMarniche D. Increasing protein at the expense of carbohydrate in the diet down-regulates glucose utilization as glucose sparing effect in rats. PloS One 6: e14664, 2011.

45. Stoger R. The thrifty epigenotype: an acquired and heritable predisposition for obesity and diabetes? Bioessays 30: 156-166, 2008.

46. Tkacs NC, Levin BE. Obesity-prone rats have preexisting defects in their counterregulatory response to insulin-induced hypoglycemia. Am J Physiol Regul Integr Comp Physiol 287: R1110-R1115, 2004.

47. Turnbaugh PJ, Ley RE, Mahowald MA, Magrini V, Mardis ER, Gordon JI. An obesity-associated gut microbiome with increased capacity for energy harvest. Nature 444: 1027-1031, 2006.

48. Wade AJ, Marbut MM, Round JM. Muscle fibre type and aetiology of obesity. Lancet 335: 805-808, 1990.

49. Warwick ZS, Weingarten HP. Determinants of high-fat diet hyperphagia: experimental dissection of orosensory and postingestive effects. Am J Physiol Regul Integr Comp Physiol 269: R30-R37, 1995.

50. West DB, Boozer CN, Moody DL, Atkinson RL. Dietary obesity in nine inbred mouse strains. Am J Physiol Regul Integr Comp Physiol 262: R1025-R1032, 1992.

51. Xie WD, Wang H, Zhang JF, Kung HF, Zhao YN, Zhang Y. Proteomic profile of visceral adipose tissues between low-fat diet-fed obesity-resistant and obesity-prone C57BL/6 mice. Mol Med Rep 3: 1047-1052, 2010.

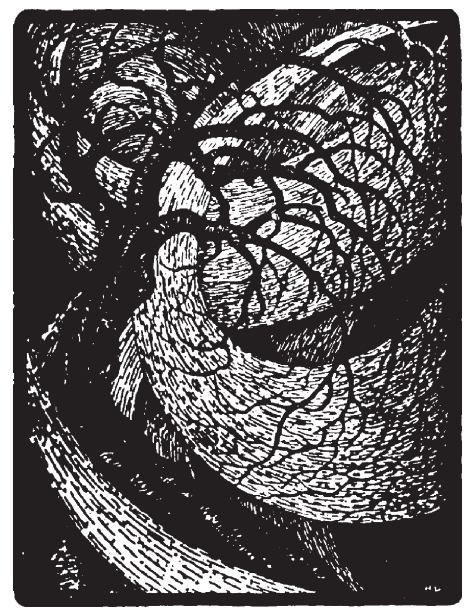

